\title{
MUL 4.0 - Digitalisierung der Wertschöpfungskette vom Rohmaterial bis hin zum Recycling
}

\author{
Manuel Woschank, Benjamin James Ralph, Alexander Kaiblinger, Philipp Miklautsch, Corina Pacher, \\ Marcel Sorger, Helmut Zsifkovits, Martin Stockinger, Stefan Pogatscher, Thomas Antretter und \\ Helmut Antrekowitsch
}

Montanuniversität Leoben, Leoben, Österreich

Eingegangen 9. April 2021; angenommen 27. April 2021; online publiziert 2. Juni 2021

\begin{abstract}
Zusammenfassung: Das Forschungsprojekt „MUL 4.0“ beschäftigt sich mit der Anwendung von Industrie 4.0 Technologien zur Digitalisierung respektive zur digitalen Transformation einer gesamten Wertschöpfungskette vom Rohmaterial bis hin zum Recycling. Der Fokus liegt hierbei auf der Erstellung von digitalen Zwillingen sowohl aus werkstoffwissenschaftlicher als auch aus produktions-technischer Perspektive. Im Rahmen der Entwicklung einer neuartigen Lernfabrik können so in realistischen Anwendungsszenarien zukunftsweisende Technologien entwickelt, getestet und verbessert werden. Zusätzlich legen neue Lehr- und Lernkonzepte den Grundstein zur nachhaltigen Entwicklung der notwendigen Schlüsselkompetenzen im Umfeld der digitalen (Lern-)Fabrik sowie zur Professionalisierung der Ingenieursausbildung der Zukunft.
\end{abstract}

Schlüsselwörter: Industrie 4.0, Lehr- und Lernfabrik, Labor, Ingenieursausbildung

MUL 4.0-Digitalization of the Value Chain from Raw Material to Recycling

Abstract: The research project "MUL 4.0" focuses on the application of Industry 4.0 technologies for the digitalization respective the digital transformation of an entire value chain from raw materials to recycling. In this context, the focus is placed on the development of digital twins, both from a materials science perspective and from a production technology perspective. As part of the development of a new type of learning factory, pioneering technologies can be developed, tested, and improved in realistic application scenarios. Moreover, new teaching and learning concepts

Dr. M. Woschank, MSc (ه)

Montanuniversität Leoben,

Franz-Josef-Straße 18,

8700 Leoben, Österreich

manuel.woschank@unileoben.ac.at lay the foundation for the sustainable development of the necessary key competencies in the environment of the digital (learning-)factory as well as for the professionalization of engineering education of tomorrow.

Keywords: Industry 4.0, Teaching and learning factory, Laboratory, Engineering education

\section{Einleitung}

Im Rahmen des Projektes "MUL 4.0" wird, basierend auf der vorhandenen Forschungsinfrastruktur an der Montanuniversität Leoben (MUL), die gesamte Wertschöpfungskette eines mehrstufigen Produktionsprozesses vollständig abgebildet und in weiterer Folge detailliert realwissenschaftlich untersucht. Basierend auf den Implikationen von einschlägigen Strategiepapieren der Europäischen Kommission, die im Wesentlichen darauf beruhen, die Wettbewerbsfähigkeit von Unternehmen als mikro-ökonomische Entitäten durch die Implementierung von Industrie 4.0 Technologien zu erhöhen, entsteht so Österreichs erste digitale Lernfabrik im Zuge eines Kooperationsprojektes von vier Instituten bzw. Lehrstühlen, welche wissenschaftlich in zahlreichen Publikationen und im Rahmen von zwei Dissertationen aufgearbeitet wird [1-4].

Basierend auf einem techno-ökonomischen Ansatz stellt diese Lernfabrik somit eine isomorphe Abbildung einer vollständig digitalisierten Wertschöpfungskette dar und soll zudem auch noch in der Lage sein, aufgrund neuester Industrie 4.0 Technologien and Technologiekonzepten Prozesse simulationsgestützt dynamisch zu beschreiben, zu erklären und letztendlich zu prognostizieren. Bereits bestehende Industrie 4.0 Lernfabriken fokussieren sich im Wesentlichen auf die experimentelle Testung von einzelnen Basistechnologien, was dazu führt, dass diese meist nur mit erheblichem Mehraufwand in das intendierte Anwendungsfeld transferiert werden können [5-7]. 
Im Projekt "MUL 4.0“ werden daher erstmalig, im Gegensatz zu den meist isoliert wirkenden Basiskonzepten bestehender Industrie 4.0 Labore, ganzheitliche und nachhaltige Maßnahmen für eine digitale Lernfabrik abgeleitet und in den Handlungsfeldern 1) der Digitalisierung und künstlicher Intelligenz, 2) der ressourceneffizienten Produktion - orientiert an Konzepten der Kreislaufwirtschaft und 3) der Mensch-Maschine Interaktion konzipiert, implementiert und wissenschaftlich untersucht. Der entscheidende Unterschied zu anderen Lernfabriken ist auch, dass hierfür keine neue und damit kostenintensive Infrastruktur angeschafft werden muss. Verwendet werden ausschließlich Maschinensysteme, welche bereits an der MUL an den beteiligten Instituten im Einsatz sind. Aufgrund einer Digitalisierungsinitiative am Lehrstuhl für Umformtechnik sind zudem ein Großteil der benötigten Betriebssysteme und Schnittstellen bereits implementiert, und es bedarf auch hier keiner groß angelegten Adaptierung. Des Weiteren ist diese dezentralisierte, digitale Lernfabrik so konzipiert, dass sie beliebig um weitere Funktionalitäten und Prozessrouten erweitert werden kann, was das Einbeziehen weiterer Lehrstühle, Studienrichtungen und Fachbereiche möglich macht. Neben dem Entstehen eines Leuchtturmprojektes wird somit auch die transdisziplinare Zusammenarbeit zwischen den verschiedensten Fachbereichen gefordert [8, 9].

\section{Das Projekt "MUL 4.0"}

Abb. 1 zeigt den Prozessablauf der MUL 4.0 Lernfabrik.

Startpunkt der digitalen Lernfabrik ist die Stranggussanlage am Lehrstuhl für Nichteisenmetallurgie (NEM). Hier wird eine zuvor spezifizierte Aluminiumlegierung mit variierenden Prozessparametern und Geometrien abgegossen sowie für nachfolgende Prozessschritte vorbereitet. Von Beginn des Gießprozesses an erfolgt zudem die durchgängige Identifikation bzw. die automatisierte Datenerfassung durch das übergeordnete Trackingsystem, welches die Produkt-, Prozess- sowie Logistikdaten (Ort, Durchlaufzeit, etc.) verarbeitet und Informationen an die nachfolgenden Prozessschritte weitergibt. Das bearbeitete Werkstück wird daraufhin vom NEM zum Lehrstuhl für Umformtechnik (LUT) weitertransportiert, wo es zunächst homogenisiert und vorgewärmt und dann in zwei oder mehreren folgenden Umformprozessen in seine Endform gebracht wird. Der Transport selbst vom NEM zum LUT wird in Echtzeit mittels der bereits erwähnten Software erfasst. Die maschinenbaulich-, metallurgisch und werkstoffwissenschaftlich wichtigsten Prozessschritte werden hierbei mittels Finite Elemente Simulation in Echtzeit abgebildet. Auf Basis dieser FE Simulation sollen Echtzeitadaptionen für das aktive Eingreifen in diese Prozesse möglich sein. Basierend auf den realphysikalischen Daten werden anschließend Digitale Zwillinge (DZ) entwickelt. Hierbei wird zudem auf den Einfluss der Thermokinetik (TK) geachtet, was zu einer besonders großen Aussagekraft der DZ führen soll. Letztere werden mithilfe von entsprechendem Qualitätsmanagement (QM) am NEM und LUT durchgeführt, validiert und kalibriert. Die adaptierten Prozessdaten werden wiederum vollständig automatisiert und kontinuierlich an ein am LUT bereits implementiertes Supervisory Control and Data Aquisition (SCADA) System übermittelt, vorbearbeitet und dann in das übergeordnete Trackingsystem transferiert. Nach Durchführung der statistischen Qualitätskontrolle können die fertigen Bauteile einer weiteren Verwendung zugeführt werden. Während beinahe aller Prozessschritte kommt es zu Materialabfall, welcher ebenfalls vollständig getrackt vom Entstehungsort systematisch an den NEM rückgeführt wird. Unter Einbeziehung von finalen Bauteilen als Altschrott schließt sich der Kreislauf der Wertschöpfungskette vollständig. Neben dem Trackingsystem werden zusätzliche Simulationen im Bereich der Logistikprozesse durchgeführt, um so laufend Bestände, Durchlaufzeiten, Termintreue und Maschinenauslastung optimieren zu können. Zusätzlich kann aufgrund des Systemverhaltens eine ideale Instandhaltungsstrategie systematisch abgeleitet werden. Die dafür eingesetzte diskrete Eventsimulation kann zudem dreidimensional dargestellt werden und ist, in Kombination mit modernen Augmented Reality Technologien, somit ein wichtiger Bestandteil der weiterführenden Lehre. Hauptverantwortlich für dieses Abbild der digitalen Lernfabrik sowie die Implementierung, Wartung und Optimierung des Tracking- und Condition-Monitoring-Systems ist hierbei der Lehrstuhl für Industrielogistik $[9,10]$.

\section{Aufgaben des Arbeitsbereichs „Umform- technik"}

Der Lehrstuhl für Umformtechnik zeichnet sich hauptverantwortlich für die Schnittstelle Manufacturing Execution System (MES)-Maschine/Prozess. Beginnend bei der Auswahl geeigneter Sensortechnik bis hin zur Implementierung geeigneter und robust agierender Machine Learning Algorithmen, werden am Lehrstuhl für die umformtechnischen Hauptaggregate geeignete Schnittstellen designt,

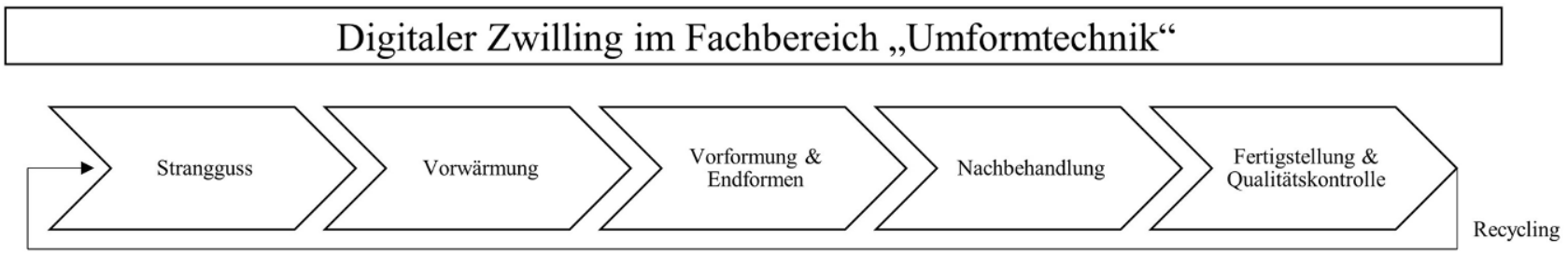

Digitaler Zwilling im Fachbereich „Industrielogistik“ 
die die Abstraktion von physikalischen Modellen zu den benötigten Logistikparametern abdecken. Die zwei Umformmaschinen, welche das vom Lehrstuhl für Nichteisenmetallurgie erhaltene Rohmaterial in die gewünschte Form bringen, sind das Versuchswalzwerk (für die Nachbildung von Blechumformprozessen) sowie die hydraulische Presse, welche für Massivumformprozesse eingesetzt wird. Für das Nachbilden praxisnaher Wärmebehandlungsrouten sowie thermomechanischer Umformung wird zusätzlich ein bestehender Ofen digitalisiert und in das Netzwerk eingebracht. Hierfür werden diese Aggregate von proprietären stand-alone Lösungen zu Cyber Physical Production Systems (CPPS) transformiert. Dabei wird besonders auf die Anwendung resilienter und kosteneffizienter Hard- und Software geachtet. Neben einem Low-Cost SCADA System kommt daher für die weitere Datenverarbeitung Python zum Einsatz, welches als Datenverarbeitungs-, Datendistributions- und Machine Learning Tool verwendet wird. Diese Algorithmen basieren auf einem Grey Box Modellierungsansatz, wobei in diesem Fall initiale realphysikalische Logiken (White Box Modelle) unter Zuhilfenahme von statistischen Methoden (Black Box Modelle) zu hybriden (Grey Box) Modellen erweitert werden. Diese Vorgehensweise sorgt für eine signifikante Reduktion der benötigten experimentellen Versuche, bei gleichzeitiger Minimierung der Gefahr des Overfittings. Die zum Einsatz kommenden Algorithmen sind hierbei besonders benutzerfreundlich aufgebaut, um für interessierte Studierende und potenzielle Industriepartner Nachvollziehbarkeit zu gewährleisten. Zusätzlich wurden für jedes CPPS zwei Front-End Visualisierungen entwickelt, welche das Verständnis für die dahinterliegende Logik steigern und die Anwendbarkeit der Lösungen in der Praxis demonstrieren. Für den Konnex der entwickelten CPPS mit einem übergeordneten Cyber Physical Logistic System (CPLS) und damit der Vernetzung der gesamten Wertschöpfungskette, wurde ein weiterer Algorithmus im Python Environment entwickelt, welcher die Anzahl der erhaltenen Daten je CPPS auf die notwendigen Parameter für die interne Logistik abstrahiert [9].

\section{Aufgaben des Arbeitsbereichs „Industrie- logistik}

Da im Zuge des Projektes die gesamte Wertschöpfungskette digital abgebildet werden soll, ist eine ganzheitliche Betrachtung der aggregats- und lehrstuhlübergreifenden Material- und Informationsflüsse von zentraler Bedeutung. Die Basis dessen legt eine möglichst frühe Kopplung der beiden Flüsse, welche durch eine Material Tracking Lösung zur eindeutigen Identifikation und Rückverfolgbarkeit der Güter vom Lehrstuhl für Industrielogistik realisiert wird. Aufbauend auf der Identifikation und Rückverfolgbarkeit der Güter wird im Zuge einer Dissertation an einer nahezu-EchtzeitBewertung der ökologischen Einflüsse der Produkte und des Prozesses gearbeitet. Dies soll durch eine low-cost Internet of Things (IoT)-Infrastruktur ermöglicht werden, welche generisch auf weitere Fertigungen ausgerollt werden kann. Die Ergebnisse dienen in weiterer Folge einem auf Primärdaten basierenden Life Cycle Assessment (LCA) und sol- len ökologische Schwachstellen des Prozesses aufzeigen. Einerseits soll als Ergebnis der Forschung ein Framework zum Ausrollen der loT-Infrastruktur in produzierenden Unternehmen vorhanden sein und andererseits sollen aus der LCA-Studie Implikationen für ähnliche Prozesse gezogen werden können. Eine weitere Dissertation beschäftigt sich mit der Entwicklung von Digitalen Zwillingen für logistische Zwecke. Hierbei bilden die Daten der MUL 4.0 Fertigungsumgebung die Grundlage des Digitalen Schattens. Zusätzlich soll ein Simulationsmodell geschaffen werden, welches die Funktionen und Verhaltensweisen innerhalb der Fertigung nachahmt. Über die Möglichkeit der Echtzeitinitialisierung wird eine Verknüpfung dieser beiden Komponenten geschaffen. Mithilfe dieser virtuellen Umgebung soll es ermöglicht werden, unterschiedliche Szenarien - bezogen auf die aktuelle Situation in der Fertigung - zu evaluieren und Handlungsempfehlungen abzuleiten sowie Vorhersagen über zukünftige Fertigungszustände zu treffen. Darüber hinaus bietet die virtuelle Umgebung die Möglichkeit, diverse Optimierungsalgorithmen zu implementieren und ihre Potenziale hinsichtlich logistischer Zielgrößen zu untersuchen. Da im MUL 4.0 Projekts eine klassische Werkstattfertigung betrachtet wird, wie sie oft bei Klein- und Mittelunternehmen zu finden ist, kann im Rahmen des Projekts insbesondere auf Anforderungen und Problemstellungen bei der Implementierung neuer Technologien in der Produktion und Logistik von KMUs eingegangen werden [1, 9-11].

\section{Lehr- und Lernprozesse im Kontext von MUL 4.0}

Im Rahmen des MUL 4.0 Projektes soll neben der Digitalisierung der Versorgungskette und der intensiven transdisziplinären Zusammenarbeit der an dem Projekt beteiligten Lehrstühle zeitgleich auch eine sogenannte Lernfabrik entstehen, um den Studierenden State-of-the-Art Ausbildungsinhalte und methodisch-didaktisch gelingende Lernumgebungen im Studium bieten zu können. Die Lernfabrik zielt auf die professionelle Vermittlung von zukünftigen essenziellen Fähigkeiten und Kompetenzen durch die aktive Einbeziehung der Studierenden ab. Im Rahmen dieser komplexen Lernprozesse bedarf es der Berücksichtigung einer Vielzahl von endogenen und exogenen Faktoren, wie Abb. 2 verdeutlicht [8, 12-15].

Betrachtet man die jeweiligen äußeren und inneren Einflussfaktoren auf die jeweilige Lernsituation wird deutlich, dass Lernen ein komplexer Prozess ist und viele (Kompetenz-)Elemente sowohl von außen bestimmt als auch in den Subjekten selbst verortet sind. Dementsprechend wird deutlich, dass die Lernfabrik als didaktisches Konzept in die Mikro-, Meso- und Makroebene der Universität eingebunden werden muss [8, 13-15]. Das Projekt MUL 4.0 trägt sowohl zum technologischen als auch methodisch-didaktischen Fortschritt und somit zur Professionalisierung der Montanuniversität bei. 


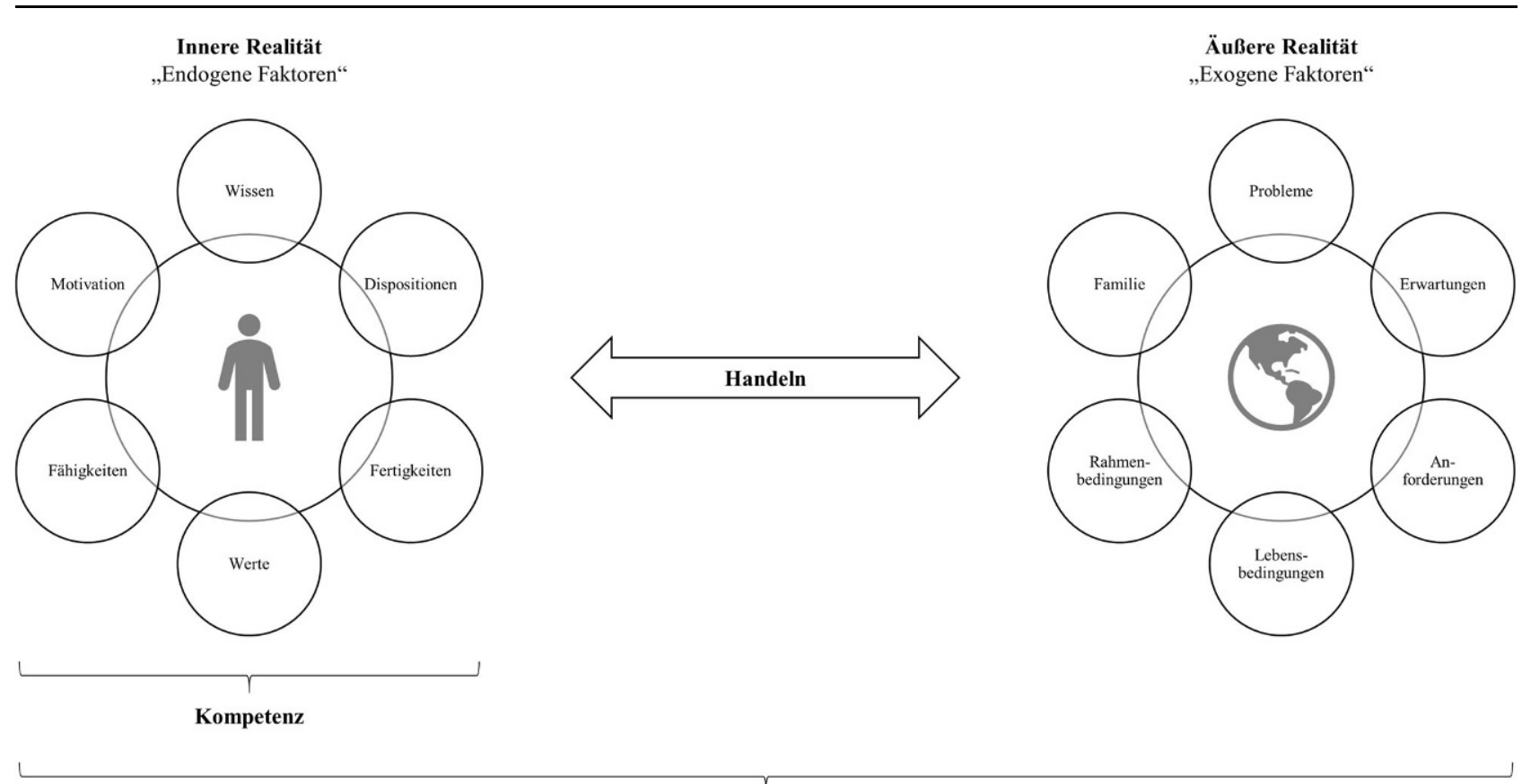

Leistung / Performance

Abb. 2: Endogene und exogene Einflussfaktoren im Lernprozess [13-15]

\section{Zusammenfassung und Ausblick}

Das innovative Forschungsvorhaben „MUL 4.0“ beschäftigt sich mit der Digitalisierung einer gesamten Wertschöpfungskette vom Rohstoff hin zum Recycling. Hierbei werden Digitale Zwillinge entwickelt deren Realitätsnähe (Validität, Reliabilität und Repräsentativität) mittels der vorherrschenden Forschungsinfrastruktur laufend überprüft und somit weiterentwickelt werden kann.

Für die dazu notwendige Digitalisierung und für die Digitale Transformation werden low-cost und open-source Lösungen implementiert, welche vor allem auf den Einsatz in KMUs bzw. bei High-Mix-Low-Volume Produktionen abzielen.

Zusätzlich sollen die dabei gewonnenen Erkenntnisse des Projekts MUL 4.0 als Fallbeispiele für Unternehmen dienen, den zweckorientierten Wissenstransfer von der experimentellen in die praktische Fertigung unterstützen sowie als Ausbildungsgrundlage für zukünftige Ingenieurlnnen zur Verfügung stehen.

Funding. Open access funding provided by Montanuniversität Leoben.

Open Access Dieser Artikel wird unter der Creative Commons Namensnennung 4.0 International Lizenz veröffentlicht, welche die Nutzung, Vervielfältigung, Bearbeitung, Verbreitung und Wiedergabe in jeglichem Medium und Format erlaubt, sofern Sie den/die ursprünglichen Autor(en) und die Quelle ordnungsgemäß nennen, einen Link zur Creative Commons Lizenz beifügen und angeben, ob Änderungen vorgenommen wurden.

Die in diesem Artikel enthaltenen Bilder und sonstiges Drittmaterial unterliegen ebenfalls der genannten Creative Commons Lizenz, sofern sich aus der Abbildungslegendenichts anderes ergibt. Sofern das betreffende Material nicht unter der genannten Creative Commons Lizenz steht und die betreffende Handlung nicht nach gesetzlichen Vorschriften erlaubt ist, ist für die oben aufgeführten Weiterverwendungen des Materials die Einwilligung des jeweiligen Rechteinhabers einzuholen.

Weitere Details zur Lizenz entnehmen Sie bitte der Lizenzinformation auf http://creativecommons.org/licenses/by/4.0/deed.de.

\section{Literatur}

1. Matt, D.T.; Modrák, V.; Zsifkovits, H. E. (eds): Industry 4.0 for SMEs, Cham: Palgrave Macmillan (Springer Nature), 2020

2. Woschank, M.; Zsifkovits, H.E.: Smart Logistics-Conceptualization and Empirical Evidence, Chiang Mai University Journal of Natural Sciences, 20 (2020), Nr. 2, S. 1-9

3. European Commission: European Skills Agenda for sustainable competitiveness, social fairness and resilience, https://ec.europa. eu/migrant-integration/librarydoc/european-skills-agenda-for-sus tainable-competitiveness-social-fairness-and-resilience (05.04.2021)

4. European Commission: A New Industrial Strategy for Europe, https://ec.europa.eu/commission/presscorner/detail/en/ip_20_416 (05.04.2021)

5. Zunk, B. M.; Bauer, U.: Bezugsrahmen „Techno-Ökonomie“: Beitrag zu einer Begriffskonkretisierung für "Technologieorientierte Unternehmen“, „Technologisch-Industrielle Leistungen“ und „Industrielle Geschäftsbeziehungen", BWL Schriftenreihe / Institut für Betriebswirtschaftslehre und Betriebssoziologie, Graz: Technische Universität, 2013

6. Kompatscher, J.; Pacher, C.; Woschank, M.: The LogiLegoLab: A Problem-based Learning Approach for Higher Education Institutions, in: Proceedings of the International Conference on Industrial Engineering and Operations Management (IEOM) Singapore, 2021, S. 1-11

7. Woschank, M.; Pacher, C.: A Holistic Didactical Approach for Industrial Logistics Engineering Education in the LOGILAB at the Montanuniversitaet Leoben, Procedia Manufacturing, 51 (2020), S. $1814-1818$

8. Ralph, B. J.; Pacher, C.; Woschank, M.: Conceptualization of the Lecture "Digitalization and Digital Transformation in Metal Forming" based on Implications from Contemporary Teaching and Learning 
Theories, in: Proceedings of the International Conference on Industrial Engineering and Operations (IEOM) Management Harare, Zimbabwe, 2020, S. 703-712

9. Ralph, B. J.; Woschank, M.; Kaiblinger, A.; Miklautsch, P.; Pacher, C.; Sorger, M.: MUL 4.0: Systematic Digitalization of a Value Chain from Raw Material to Recycling, Procedia Manufacturing, 52 (2021) S. 1-8 (in press)

10. Woschank, M.; Kaiblinger, A.; Miklautsch, P.: Digitalization in Industrial Logistics: Contemporary Evidence and Future Directions, in: Proceedings of the International Conference on Industrial Engineering and Operations Management (IEOM) Singapore, 2021, S. 1-12

11. Matt, D. T.; Modrák, V.; Zsifkovits, H. E. (eds): Industry 4.0 for SMEs: Concepts, Implementation and Applications, Cham: Palgrave Macmillan (Springer Nature), 2021

12. Pacher, C.; Woschank, M.: Competencies in the Digitalized Working Environment: A Concept for Engineering Education in Higher Education Institutions, in: Proceedings of the International Conference on Industrial Engineering and Operations (IEOM) Management Harare, Zimbabwe, 2020, S. 1203-1212

13. Woschank, M.; Pacher, C.: Fostering Transformative Learning Processes in Industrial Engineering Education, in: Proceedings of the 5th NA International Conference on Industrial Engineering and Operations Management (IEOM) Detroit, Michigan, USA, 2020, S. 2022-2029

14. Woschank, M.; Pacher, C.: Program Planning in the Context of Industrial Logistics Engineering Education, Procedia Manufacturing, 51 (2020), S. 1819-1824

15. Woschank, M.; Pacher, C.: Teaching and Learning Methods in the Context of Industrial Logistics Engineering Education. Procedia Manufacturing, 51 (2020), S. 1709-1716

Hinweis des Verlags. Der Verlag bleibt in Hinblick auf geografische Zuordnungen und Gebietsbezeichnungen in veröffentlichten Karten und Institutsadressen neutral. 